\title{
Rejoinder on: Tests for multivariate normality-a critical review with emphasis on weighted $L^{2}$-statistics
}

\author{
Bruno Ebner $^{1} \cdot$ Norbert Henze $^{1}$ (D)
}

Received: 28 October 2020 / Accepted: 30 October 2020 / Published online: 1 December 2020

(c) The Author(s) 2020

First of all, we thank all of you for your most valuable comments regarding our manuscript!

Professor Jiménez-Gamero, in particular, comments on the energy test in connection with the class of BHEP tests and on testing normality for non-observable data originating from i.i.d. data. We found it very interesting to see that the energy test statistic of Székely and Rizzo belongs to the class of BHEP statistics which, in spirit of results of Baringhaus et al. (2017), shows that the energy test statistic, after a suitable affine transformation, has a normal limit under fixed alternatives. Such a result answers a question that we posed in our review. We also thank Prof. Jiménez-Gamero for pointing out results and open problems in connection with testing for normality for the distribution of errors in linear models or innovations in MGARCH models. In these cases, tests are based on residuals, which are not i.i.d. The authors will use the input of Professor Jiménez-Gamero regarding these models to update the description of the accompanying R package mnt, see Betsch and Ebner (2020).

Professor Meintanis draws attention to the issue of computation time, which is becoming more and more important in view of nearly ubiquitously present highdimensional data. He points out that, in order to obtain a representation of a weighted $L^{2}$-statistic that is free of integrals, when testing the goodness of fit of an $\alpha$-stable distribution, the weight function should be a suitable spherical Kotz-type density. The underlying concept is that of adjoint distributions, where the density of one distribution is a multiple of the characteristic function of the other distribution.

\footnotetext{
This rejoinder refers to the comments available at: https://doi.org/10.1007/s11749-020-00739-7, doi: https://doi.org/10.1007/s11749-020-00741-z, https://doi.org/10.1007/s11749-020-00742-y, https://doi.org/10.1007/s11749-020-00743-x.
}

$\bowtie$ Norbert Henze

norbert.henze@kit.edu

Bruno Ebner

bruno.ebner@kit.edu

1 Institute of Stochastics, Karlsruhe Institute of Technology (KIT), Englerstr. 2, 76133 Karlsruhe, Germany 
Professor Richards raises a variety of intriguing research questions. These refer to tests for normality in the case of incomplete data (notably an extension of the results of Yamada et al. (2015) to obtain kurtosis tests) and the option of having $L^{p}$-tests (where $p$ could even be estimated from the data), but also to ascertain the value of recent kernel Stein discrepancy methods for $L^{2}$-tests for normality. Regarding the latter point, it is noteworthy that the recent test of Ebner et al. (2020) is motivated by a multivariate Stein equation and, as mentioned in that paper, such a motivation also holds for the test of multivariate normality in Henze and Visagie (2020). It is obvious that similar ideas could be used for a wide variety of parametric families of univariate and multivariate distributions, since Stein's method of approximation has been extended to many other families of distributions, for characterizations of distributions related to this subject see Betsch and Ebner (2019) and the references therein.

Moreover, Professor Richards asks whether the integral representation of the BHEP statistic given at the beginning of Section 3 can be extended to non-normal distributions. Up to now, we do not have an answer to this interesting question.

As for the comment regarding a simple explanation on why the functional $T_{\mathrm{CS}}$ vanishes on the set $\mathcal{N}_{d}$, we consult Ebner (2012) to see that the test of Cox and Small was motivated by the wish to assess the occurrence of nonlinearity of dependence in the underlying population. Consequently, the test statistic uses the regression coefficient of the quadratic component in a suitably chosen regression model on linear combinations of the components of the random vectors. Since the normal distribution is uniquely determined by the mean vector and the covariance matrix, it merely displays linear relationships between the components of the normal distributed random vector, and hence, the functional vanishes. One can easily see that the latter property also holds certain non-normal distributions satisfying a weak moment condition, such as elliptically symmetric distributions.

The question of obtaining results on the behavior of the energy test with respect to contiguous alternatives seems to be solved in view of the observation of Professor Jiménez-Gamero that the energy statistic is a special case of the class of BHEP tests. Finally, there is the fundamentally important problem to obtain explicit expressions for the eigenvalues and the corresponding eigenfunctions associated with the Fredholm integral equations that pertain to weighted $L^{2}$-statistics. In this respect, Professor Richards makes a valuable list of some cases in which such explicit expressions have been obtained. The case of the BHEP statistic, however, is still open.

Professors Rizzo and Székely elaborate on a promising generalization of the energy test, which is based on a so-called $\alpha$-energy distance, where $0<\alpha<2$. At the end of their contribution, the authors raise the natural question whether there is an 'optimal' way to choose the parameter $\alpha$ in order maximize the power of the tests of normality. A first step in this direction would be an extensive simulation study, for which the accompanying R package mnt, see Betsch and Ebner (2020), may be of benefit. On a more theoretical level, the methods proposed by Tenreiro (2019) are noteworthy. Although they are formulated in the univariate setting, they can be straightforwardly extended to the multivariate case. 
Acknowledgements The authors thank the editor for the invitation to write this survey and two anonymous referees for many constructive comments. We are especially grateful to the four discussants, whose contributions put the topic into a broader perspective and will certainly stimulate further research.

Funding Open Access funding enabled and organized by Projekt DEAL.

Open Access This article is licensed under a Creative Commons Attribution 4.0 International License, which permits use, sharing, adaptation, distribution and reproduction in any medium or format, as long as you give appropriate credit to the original author(s) and the source, provide a link to the Creative Commons licence, and indicate if changes were made. The images or other third party material in this article are included in the article's Creative Commons licence, unless indicated otherwise in a credit line to the material. If material is not included in the article's Creative Commons licence and your intended use is not permitted by statutory regulation or exceeds the permitted use, you will need to obtain permission directly from the copyright holder. To view a copy of this licence, visit http://creativecommons.org/licenses/by/4.0/.

\section{References}

Baringhaus L, Ebner B, Henze N (2017) The limit distribution of weighted $L^{2}$-statistics under fixed alternatives, with applications. Ann Inst Stat Math 69(5):969-995

Betsch S, Ebner B (2019) Fixed point characterizations of continuous univariate probability distributions and their applications. Ann Inst Stat Math. https://doi.org/10.1007/s10463-019-00735-1

Butsch L, Ebner B (2020) mnt: Affine invariant tests of multivariate normality. R package version 1.3. https://CRAN.R-project.org/package $=m n t$

Ebner B (2012) Asymptotic theory for the test for multivariate normality by Cox and Small. J Multivar Anal 111:368-379 ISSN 0047-259X

Ebner B, Henze N, Strieder D (2020) Testing normality in any dimension by Fourier methods in a multivariate Stein equation. arXiv preprint arXiv:2007.02596

Henze N, Visagie J (2020) Testing for normality in any dimension based on a partial differential equation involving the moment generating function. Ann Inst Stat Math 72(5):1109-1136

Tenreiro C (2019) On the automatic selection of the tuning parameter appearing in certain families of goodness-of-fit tests. J Stat Comput Simul 89(10):1780-1797

Yamada T, Romer MM, Richards DSP (2015) Kurtosis tests for multivariate normality with monotone incomplete data. TEST 24(3):532-557

Publisher's Note Springer Nature remains neutral with regard to jurisdictional claims in published maps and institutional affiliations. 